\title{
Effect of changing the saddle angle on the incidence of low back pain in recreational bicyclists
}

Moshe Salai, Tamar Brosh, Alexander Blankstein, Arial Oran, Aharon Chechik

\begin{abstract}
Objective-According to the literature, $30-70 \%$ of cyclists suffer from cervical, dorsal, or lumbar back pain. This study was conducted to evaluate one of the possible causes of low back pain and to suggest a solution by appropriate adjustments to the bicycle.

Methods-Serial fluoroscopic studies were performed while cyclists sat on different types of bicycle (sports, mountain, and city). Pelvic/spine angles were measured at different seat angles, and the related force vectors analysed.

Results-There was a tendency towards hyperextension of the pelvic/spine angle which resulted in an increase in tensile forces at the promontorium. These forces can easily be reduced by appropriate adjustment of the seat angle-that is, by creating an anterior inclining angle. The findings of the biomechanical analysis were then applied to a group of cyclists who were members of a cycling club and who complained of low back pain. After appropriate adjustment of the saddle angle, most of the cyclists $(>70 \%)$ reported major improvement in the incidence and magnitude of their back pain.

Conclusions-The incidence and magnitude of back pain in cyclists can be reduced by appropriate adjustment of the angle of the saddle. It is important that these findings be conveyed to cyclists, bicycle salesmen, trainers, and members of the general public who engage in cycling, in order to decrease the prevalence of back pain.
\end{abstract}

(Br F Sports Med 1999;33:398-400)

Keywords: cycling; back pain; saddle angle
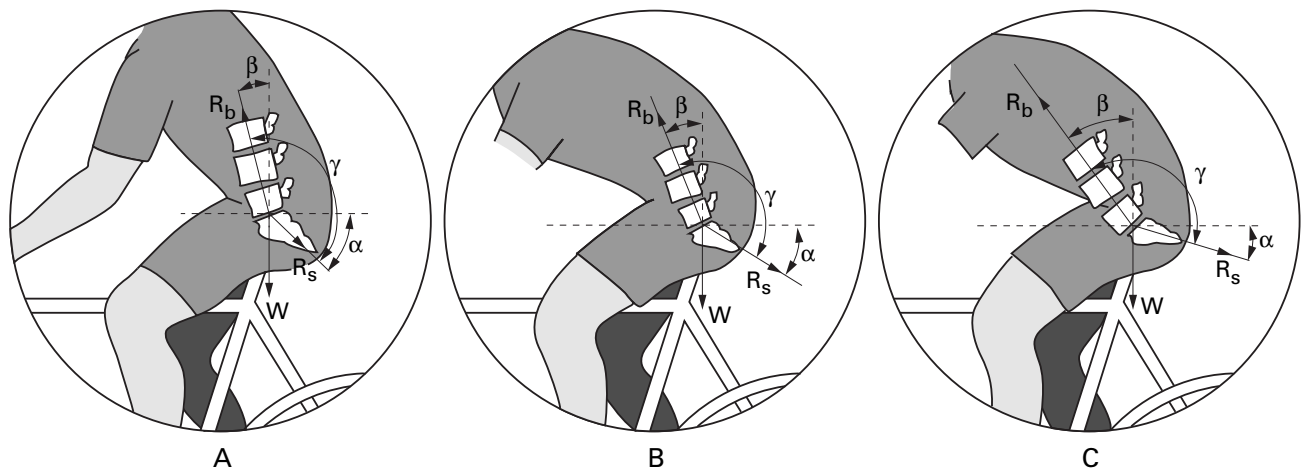

Figure 1 Lateral pelvic/spine schema drawn from radiographs taken while the subject was sitting on various bicycles with various body positions, showing the related force vectors at the promontorium. $W$, weight; $R_{b}$, lumbar vector; $R$, pelvic vector; $a$, angle between ground and $R_{s}$ vector; $\beta$, angle between weight axis and $R_{b}$ vector; $\gamma$, lumbosacrallpelvic angle. $(A)$ Town bike; (B) mountain bike; $(C)$ racing bike.
Cycling is one of the most popular recreational sports, and also an important means of transport for millions throughout the world. Various publications have reported on back pain at various levels of the spine among cyclists at rates of 30-70\%. It often causes recreational cyclists to abandon the sport, but for many others (such as in China), cycling is their only means of urban transportation. ${ }^{1-5}$

The forces acting on the anterior lumbar spine in the sagittal plane are mostly tensile. Very few studies in the orthopaedic and sport medicine literature have investigated the causes of back pain in amateur cyclists, and no methods have been proposed for reducing the incidence of back pain in cyclists. ${ }^{6-9}$ This study concentrates on biomechanical evaluation only of the forces involved in the lower back during cycling. The results of the biomechanical analysis were then applied to different types of bicycle, which resulted in major clinical improvement.

\section{Materials and methods}

A fluoroscopic study was conducted in which position of cyclists were taken while they were sitting on different types of bicycle: city, mountain, and racing. Ten healthy adults were randomly elected for each type of bicycle, and were analysed by fluoroscopy. The pelviclumbar spine angles were measured for each and each type in relation to the ground level (fig 1).

The effects of changing the sitting position and angle of the saddle on the vectors acting at the promontorium (as a reference point) were recorded (fig 2). This indicated that increasing the $\alpha$ angle, namely the anterior saddle inclination, reduces the tensile pelvic and lumbar vectors (table 1). images of the lumbosacral region in the lateral

M Salai

A Blankstein

A Oran

Laboratory for Exact Measurements, School of Dental Medicine, Sackler School of Medicine, Tel Aviv University, Israel $\mathrm{T}$ Brosh

Dr M Salai, Department of Orthopedic Surgery A, The Chaim Sheba Medical 52621, Israel.

Accepted for publication 7 September 1999 


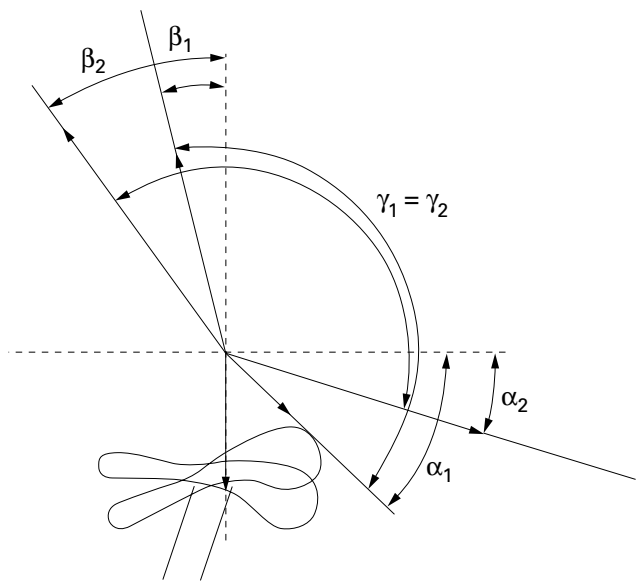

Figure 2 Changing the seat angle from horizontal to an anterior inclination causes the $a$ angle to decrease $\left(\alpha_{2}<a_{1}\right)$; the $\gamma$ angle remains constant $\left(\gamma_{1}=\gamma_{2}\right)$ and the $\beta$ angle increases $\left(\beta_{2}>\beta_{1}\right)$.

Table 1 Effect of a angle on the $R_{b}$ to $R_{s}$ ratio

\begin{tabular}{rlll}
\hline$a$ & $R_{b} / R_{s}$ & $R_{s}(W)$ & $R_{b}(W)$ \\
\hline 0 & 1.22 & 1.43 & 1.74 \\
10 & 1.39 & 1.24 & 1.72 \\
20 & 1.64 & 1.00 & 1.64 \\
30 & 2.05 & 0.74 & 1.52 \\
40 & 2.96 & 0.45 & 1.33
\end{tabular}

$\alpha$ angle, the angle between the saddle and ground; $R_{b} / R_{s}$, tensile vectors acting on the promontorium: $R_{b}$, upward vector; $R_{s}$, downward vector.

After these preliminary studies, 80 members of a cycling club were questioned about their back pain. The group consisted of 30 women and 50 men, whose ages ranged from 17 to 72 years, and who cycled between 30 and $80 \mathrm{~km}$ a week on mountain/unpaved trails. They comprised a group of "occasional" cyclists who were not involved in competitions, and who usually cycled during weekends as a recreational sports activity. Thirty four of them rode sport/racing bicycles, 31 mountain bicycles, and 15 city bicycles. Their average biking distances a week were: $30-50 \mathrm{~km}(20 \%), 50-60$ $\mathrm{km}(25 \%)$, and $60-80 \mathrm{~km}(55 \%)$. Forty members $(50 \%)$ reported the occurrence of low back pain of intermediate intensity (necessitating the use of minor analgesics and a few days of rest after cycling). Twenty five of these rode sports bicycles, and 15 rode mountain bicycles. The results of the fluoroscopy and its biomechanical analysis were applied to the bikes of these $40 \mathrm{club}$ members - that is, the seats were adjusted to produce an anterior angle of inclination ( $\alpha$ angle of $10-15^{\circ}$ ). The angle was set using an orthopaedic goniometer, adjusted for each bicycle. The decision to use either a $10^{\circ}$ or a $15^{\circ}$ angle was arbitrary. Each group comprised 20 cyclists. An angle greater than $15^{\circ}$ caused discomfort and resulted in the rider slipping from the saddle. All the cyclists were followed up and questioned again about their back pain after six months.

\section{Results}

BIOMECHANICAL ANALYSIS

The fluoroscopic and biomechanical analysis studies showed that, in order to maintain equilibrium while cycling, an equilibrium in both the horizontal (X) and vertical (Y) direction should be kept at all times-that is, $\Sigma \mathrm{X}=0 ; \mathrm{R}_{\mathrm{b}} \sin \beta-\mathrm{R} \cos \alpha=0$ and $\Sigma \mathrm{Y}=0$; $\mathrm{R}_{\mathrm{b}} \cos \beta-\mathrm{R}_{\mathrm{s}} \sin \alpha=0$

Thus an increase in the $R_{b}$ to $R_{s}$ ratio is directly related to the $\alpha$ angle (fig 1 , table 1 ). Theoretically, a similar effect of reducing the tensile forces acting on the promontorium could be achieved by elevating the saddle tip. However, practically this is impossible, as it causes the rider to slip off the saddle thereby producing pressure on the perineum and genitalia. It is also clear from this analysis that this angle is smaller when the rider leans forward on a racing bike, relative to the angle on a town bike; however, the aerodynamic position and resistance are different (these factors are beyond the scope of this study).

CLINICAL TRIAL

After six months in which no change in cycling habits occurred, the 40 cyclists were reevaluated by personal interview about their back pain. Twenty nine $(72 \%)$ reported that they no longer had back pain, eight $(20 \%)$ reported major reduction in the occurrence and magnitude of the pain, and three $(7 \%)$ reported no change. A $t$ test was used for comparison of continuous variables with Yates correction for comparison of binary variables. Using the existence of low back pain as a variant, these results were also statistically valid $(p<0.01)$. No statistically valid correlation was found with type of bicycle, gender, age, distance cycled a week, or the angle of inclination $\left(10^{\circ}\right.$ or $\left.15^{\circ}\right)$.

\section{Discussion}

Throughout the world, millions of people ride bicycles as an aerobic form of recreational sport which does not demand special skills or complex equipment and has no age limits. Others, from children to the elderly, ride bicycles as a means of cheap daily transportation which does not cause air pollution or traffic jams.

The occurrence of back pain resulting from bicycling is probably far higher than has been emphasised in orthopaedic or sports medicine literature. ${ }^{3}$ Many factors affect the vectors acting in the body during cycling, such as height of the saddle and handle bars, distance between saddle and handle bars, type of bicycle, frame size, type and height of the saddle, length of cranks, individual morphology, gearing, and cadence. In this study, we concentrated on the saddle angle only, a subject previously not discussed, yet with major effects on the vectors at the lumbopelvic region.

From our fluoroscopic/biomechanical study of cyclists, it appears that low back pain can be attributed, in part, to the anatomical extension between the pelvis and the spine. This results in tensile forces along the anterior longitudinal ligament of the lumbar spine, which increase as the result of sitting on the saddle and reclining on the handle bar, as has been shown in this work.

Proper adjustment of bicycles should be the result of correct definition of the purpose of their use. Often there are contradictions 
between the different demands of cycling - for example, speed versus comfort. This is partially due to the fact that most energy is expended during cycling to overcome the friction of the cyclist and bicycle against the air. ${ }^{10-12}$ Another major factor is the relation between cadence and gears. Thus, when using racing or triathlon bicycles, one "pays the price" of decreased air resistance by having to assume a very low profile and leaning non-physiologically on an aerobar to achieve greater speed.

At the other end of the scale are the urban/city bicycles where an upright sitting position (although they have high air resistance) results in lower speed, yet lower tensile vectors at the lumbar region, and more options for proper adjustment of the bicycles. Therefore it is incumbent on doctors to alert recreational cyclists and the public to the potential hazards to the lower back of riding inappropriately adjusted bicycles. Low back pain among cyclists is sometimes self limiting, with natural remission. However, the statistically valid results of our studies are highly convincing with regard to the efficiency of reducing the occurrence of back pain by adjusting the saddle angle. As shown in our study, an anterior $10-15^{\circ}$ inclination angle of the saddle is one early important step that can be taken.

Bicycle salesmen should be requested to routinely use the "fit-kit" (a table used by some manufacturers with recommendations on, among others, the suggested height of the saddle and saddle-handle bar distance adjustments for specific bicycles) when selling bicycles, after inquiring about the purpose of the purchase. This is most important for a growing child in whom long hours of increased hyperextension between the spine and pelvis may interfere with proper growth and produce future back pain. Further biomechanical studies are necessary to evaluate other previously mentioned parameters, such as the biomechanical effects of the saddle height/bar distances. The results of this and further studies should then be presented to bicycle manufacturers, with a demand that all bicycles be produced with options to adjust these parameters on purchase. Adjustment, as shown in our study, could reduce the prevalence of back pain in cyclists, and result in healthier and more pleasurable involvement in this popular sport.

1 Barry D, Weiss BD. Nontraumatic injuries in amateur long distance bicyclists. Am f Sports Med 1985;13:187-92.

2 Mayer PJ. Helping your patients avoid bicycling injuries. $\mathcal{F}$ Musculoskeletal Medicine 1985;2:31-8.

3 Mellion MD. Neck and back pains in bicycling. Clin Sports Med 1994;13:137-63.

4 Mellion MB. Common cycling injuries. Management and prevention. Sports Med 1991;11:52-70.

5 Pfeiffer RP, Kronisch RL. Off road cycling injuries. An overview. Sports Med 1995;19:311-25

6 Ryschon TW, Gundersen J. The effect of body position on the energy cost of cycling. Med Sci Sports Exerc 1991;23: $949-53$.

7 Stone C, Hull ML. The effect of rider weight on rider-induced loads during common cycling situations. $f$ Biomech 1995;28:365-75.

8 Too D. Biomechanics of cycling and factors affecting performance. Sports Med 1990;10:286-302.

9 Yoshihuku Y, Herzog W. Optimal design parameters of the bicycle-rider system for maximal muscle power output. $\mathcal{F}$ Bicycle-rider system for max

10 Cappelli C, Rosa G, Butti F. Energy cost and efficiency of riding aerodynamic bicycles. Eur $\mathcal{F}$ Appl Physiol 1993;67: 144-9.

11 de Groot G, Welbergen E, Clijsen L. Power, muscular work, and external forces in cycling. Ergonomics 1994;37:31-42.

2 Kyle CR. Energy and aerodynamics in bicycling. Clin Sports Med 1994;13:37-46.

Take home message

Low back pain is common among cyclists, regardless of age, gender, or type of bicycle. Its incidence and severity can be reduced by adjusting the saddle to introduce a $10-15^{\circ}$ anterior inclination. 